\title{
TQM in Healthcare: Transformation, Challenges, Impact and Measurement
}

\author{
Agadeer Mutahar $^{1}$, Bayan AlDoaies ${ }^{2}$, and Azrilah Abdulaziz ${ }^{3}$ \\ Information Systems Department, Faculty of Computing \& Information Technology, King Abdulaziz University, \\ Jeddah, $\mathrm{KSA}^{1,2}$ \\ $\mathrm{PhD}$, Information Systems Department, Faculty of Computing \& Information Technology, King Abdulaziz University, \\ Jeddah, $\mathrm{KSA}^{3}$
}

\begin{abstract}
The critical success key of healthcare will lie in its ability to enhance the quality of patient care. Over the last view years, the computerized information system applied in the healthcare setting to increase the quality degree. Also, providing services for health individual and population to increase the probability of required outcomes. In this paper, we made an overview of healthcare information system (HCIS) from the total quality management (TQM) aspect. Therefore, the research includes the transformation challenges, the effect of HCIS on the healthcare quality, and what measurement used to assess the quality level of a healthcare environment. Consequently, the using of HCIS in the healthcare organization activities will lead to raising and improving efficiency, accomplish its goals and achieve an elevated level of quality.
\end{abstract}

Keywords: Healthcare, Total Quality Management (TQM), Computerized, Healthcare information system (HCIS), Outcomes, and Measurement.

\section{INTRODUCTION}

No one hides the nature of the current situation from the aggressive competition observed by the healthcare sector, include services variety of methods and techniques. Moreover, the movement of change accelerates in an unprecedented way, which makes the healthcare organization and hospital seeks to permanent searching to ensure their position in the healthcare environments. As a result, several concepts have emerged to accompany this competitive environment, and these concepts constitute a means of entry and continuity in the world of competition strongly. Thus, the importance of the system increased, health organization strived to establish a Total Quality Management. This paper tackles the issue of the importance of information system and its effect on the total quality management of health services. Furthermore, the Total Quality Management (TQM) is the ability to produce a commodity or service to meet the needs of the consumer, it is a variable of measurement according to the specifications and predetermined by specialists. Additionally, the total characteristics of the patient care services that appear in the ability of meeting the patient needs to satisfy them. Finally, the process of achieving quality becomes a goal, to define what is a healthcare information system. This paper will discuss this concept and it is components. The information system is a processing result of the data and used to decision making, disease prevention, and treatment. Furthermore, the system is a combination of input, output, and processing which organized and based on each other. Consequently, information system (IS) defines as a set of integrated components to facilitate the control, communication, and decision-making to achieve organization objectives. Therefore, the healthcare information system (HCIS) indicates the computerized information system applied in healthcare setting [26]. This paper aims to make an overview of the history and current status of HCIS in health organizations. Also, it discusses the transformation challenges from the old system to the current one. Moreover, we seek to review the impact of TQM in information system improvement and ways to measure the system quality. Also, with providing some examples of HCIS to take its advantages and deploy it. Finally, the paper provides recommendations for HCIS improvement and how we can make it smarter and aligned with the TQM environment. Moreover, many researchers applied a contribution to enhance the total quality of healthcare by using the information system. Through evaluate the IS on healthcare, and illustrate some instance of IS to improve the TQM. The data quality effected on healthcare system was the key point which discussed by [7]. by studied the impact based on three essential points. First, the effect of complex information and errors that appear in the healthcare system. Second, the relating data quality issues and quality assurance. Finally, suggestion six models to present the quality of the data in healthcare system. Moreover, to improve the quality of the data, the stockholder must provide a training to avoid any errors, insure from the original data that patient provided to take a right treatment decision, and change the culture to patient safety. Besides, [20] described how TQM of IS a newest in healthcare area, founding integrated information with ability to acceptable and elaborate the quality strategies. Then, they proposed a model that describe how IS 


\section{IARJSET

designed the healthcare system in TQM environment. listed the key concept to implement TQM in the healthcare system which are corporate framework created for quality, change corporate culture, end user focusing, improvement process by collaborate approach, education and training level for the employees. Also, it contains the top of leadership management, statistical report, benchmarking, and quality measurement. Moreover, the evaluation of IS in healthcare and what problems acting as barriers for quality improvement are topics that discussed by [1] and [26]. First [1], defined all problems and recommended solutions for apply the IS in a healthcare to reduce the clinical errors. As well, [26] investigate in evaluation of health information systems. In their study, they illustrated different HCISs and classify it depending on its descriptions and characteristics. The evaluation for HIS based on comprehensive and specific measures. Furthermore, the researchers [5] described the transformation from manual to system transaction in a healthcare. Then, they discussed the success and failed factors for implementing information system at healthcare. Also, they described the importance of participate the end user in designed process to made a success system and improve its quality. Further, most of health organizations searching for ways to improve the TQM of healthcare process and measures. Wherefore, [17] proposed the quality assessment tool which is ensuring quality information for patients (EQIP). This tool assists the objective and precision of written healthcare information to ensure its reliability and validity. For the same purpose, the researchers [23] and [13] convert the traditional nursing documentation to the electronic health record system (EHR). This system aims to facilitate the process of hospitalized patients' information to enhance the TQM of patient care. [13] studied the effect of IS on the TQM at healthcare. Besides, [23] examined the EHR on multiple hospitals. Consequently, they reached that EHR system plays as a key role in quality improvement through a rapidly access to the patient's information and integration with decision support system. Also, the variant features add to the healthcare environment. The integrated electronic clinical reminder system developed by [22]. This system improved quality care for diabetes and coronary artery disease through the clinical reminders. In contrast, it has a limited impact on the total quality of healthcare. The laboratory data may be facing many difficulties through the workflow of results as, error-prone or delays in communication especially in resource-poor setting. For that, the [6] proposed an electronic laboratory information system to enhance the laboratory data quality. They noted the efficiency of the developed web-based information system in order to improve the quality of patient care and health monitoring. Besides, the researchers [4] determined the challenges of merging the IS with healthcare environment. They discussed challenges and difficulties that may appear with un-computerized care system at health environment. For instance, avoiding the health care errors, interpret and analyses the complexity tables of consultation result. However, these challenges play as key barriers to improve the total quality of healthcare. By the same token, [12] used an integrated information and decision system to improve the quality of healthcare. They studied Veterans Health Administration (VHA) system and consider it as investment to improve the research quality and compared between VHA systems with Vista. Finally, [18] explained the critical importance of information system with determined the outcomes to compare it with healthcare quality guideline. Then, they organized the informatics blueprint for healthcare information system to serve the oncology researchers. Additionally, they used the outcomes to build and evaluate healthcare system according to related area .In overall, the core purpose of all researches is to investigate probable ways to enhance the TQM improvement. Therefore, in this work we going to implement HCIS to develop the workflow in healthcare organization. Also, increase the patient and employee's satisfaction.

\section{THE CONCEPT OF TOTAL QUALITY MANAGEMENT (TQM)}

According to ISO 8402, the TQM is sometimes called "total quality", "company-wide quality control", "total quality control TQC", etc. There are many definitions for TQM concept as illustrated in the following. The ISO 8402 defines TQM as management approach of an enterprise focused on quality that involves all its members in a business from upper management to production line workers for long-term success, through client satisfaction and benefits for all members of enterprise and society [3]. Also, "All activities of the overall management function that define the quality policy, purposes and tasks, and implement them by means such as quality planning, quality control, quality assurance and quality improvement within the quality system "[16]. Moreover, looking toward a future is very significant on TQM, through a rapid changing in the world the TQM is improving the system practice, tools, methods, strategies by an organization, in order to create higher quality products, services, and reduce waste in the business, to gain customers satisfaction [3]. Additionally, Feigenbaum (1991) defined TQM as: "An effective system for integrating the qualitydevelopment, quality-maintenance, and quality-improvement efforts of the several sets in an organization to enable marketing, engineering, production, and service which allow for full customer satisfaction" [16]. As a consequence, for the mentioned definitions, the concept of TQM based on three main principles to achieve the organization aims. These principles are the continuous enhancement for each part in the organization for produce a high quality of products, services, and reduce waste in the business which leads to customer satisfaction. Applied the TQM in healthcare will enhance the healthcare services, simplifies the workflow process, gain patients' and providers' satisfaction, reduce cost, and timely delivery for valuable clinical knowledge [10]. Also, it assists the facilitating and recognizing training opportunities, engaging staff, and setting organization-wide direction [9]. Furthermore, the monitoring of healthcare activities prevents any errors that may expose the healthcare activities for risks or reduce its quality [10]. 


\section{IARJSET

\section{HEALTHCARE QUALITY IN THE PAST AND PRESENT}

In the former years, the healthcare organization used paper-based system in all internal process this way faced a lot of limitations that has impact on many aspects as cost, productivity, TQM and more. This paper will focus on total quality issues and how the manual record effect on the healthcare industry. The issues of TQM encompass the patient care, financial and documentation affairs. However, the main challenge of paper is related to the documentation issues. For instance, using the paper record may increase the exposure to risk, with the natural incidents as fire or flood. Moreover, working on the paper record not guarantees the integrity of information through loss, torn or stained documents. However, guarantee the quality of patient documentation is very significant in the health domain. The different staff documentation with different knowledge, skills, and standardization will provide different quality levels of the patient's documentation. These differences will generate an incomplete and an inconsistent healthcare data which will affect consultation treatment results and medication orders. In the same token, this consultation history records will be stored on paper archived and will not be accessible for medical staff and need the period of times to check it. Moreover, the patient information required across the hospital sections cannot always be updated instantly which moreover cause on wasting of time and an inconsistency. The patient takes a long time in the consultation process and takes much time for the hospital administrative to find patient files in the archive library. According to the technology improvement, the orientation to integrate the healthcare with information system recommended for enhancing the following issues. First of all, the ability to save and processing, retrieval and exchange the clinical information relating to patients by HCIS. The patient has an account includes his entire medical history, without the need to relist the entire medical history whenever the patient visits a new doctor. As well as, this system leads to enhance the overall quality of healthcare by minimizing the incidence of adverse drug reactions and provide more precise medical history, which assists the physician and patient to take decisions about treatment. Moreover, this organization can prepare a lot of statistics, a lot of the most common types of the disease or chronic diseases. The healthcare quality must make the decision, communicate with caregivers, and provide the alert in some situations and all features not available with the paperbased system in healthcare. Finally, the healthcare organization must provide the training session for their employees to facilitate this transformation and raise the level of healthcare quality.

\section{TRANSFORMATION CHALLENGES}

In health environment, the transformation from the old system to the newest one has a multiple challenges and barriers. According to the complexity of healthcare workflow and the need for cooperating across multiple disciplines, departments, and specialist for providing the recommended patient care, the development of health system becoming a significant issue as mentioned in the Section 4. In this Section, the focus will be on the challenges of transformation process from traditional paper-based system to the computerized information system, the significant barriers include; (i) the typical hospital used an enormous number of devices with advanced network connection and communication setting. Besides, these infrastructures require an adequate funds to pay new system, effort, and maintain a process to achieve a high scale level of the computerized system; (ii) transform the patient information between different hospital departments as pharmacy, emergency, wards or more is one of major task of HCIS. The main challenges are a lack of standards and high performing interface; (iii) taking a decision to build a single or various system for the different hospital departments. For instance, the developer should implement specialist system for each department and linked to each other to transform the patient information or should all departments share a single system; (iv) HCIS must be a robust and reliable system by reducing the failure rate, increase the availability, portability, usability, and the flexibility of the system. Also, concerns about the confidentiality and the privacy of patient records. In ensuring, to achieve these requirements, the key point is choosing the optimal IT to meet the HCIS goals; and (v) the challenges not limited to hardware/software issues only, but also the medical staff affect the transformation process. The differences of the staff expertise and background may effect in understanding the overall workflow of HCIS. Also, the fear or/and inexperience of used the new system [19]. Obviously, the key success factor for transformation process is to handle and anticipate these challenges by the organization involved. Moreover, the requirement integration and well understanding the HCIS process from the medical staff will guarantee the success of the system.

\section{AN OVERVIEW OF HEALTHCARE INFORMATION SYSTEM (HCIS)}

In recent years, the importance of using the information system in modern societies has grown to the point that we are now in the era of the information revolution, the media and healthcare systems. Further, it depends directly on the matching between advanced technology and how to manage its usages. The HCIS can define as "a set of comprehensive and integrated computerized systems designed to store, process, distribute and use information" [26]. According to the Saudi Society for Health Informatics they defined the HCIS as "a comprehensive and integrated information system used in the management of hospital and administrative that aims to enable the hospital and its staff to provide the best and fastest care for their patients" [21]. As result of those definitions, the HCIS characterized as a 


\section{IARJSET

gathering of interrelated procedures implemented to help in improving the productivity and effectiveness of a healthcare organization in executing its capacities and achieving its goals [21]. However, the process of providing patient healthcare that requires high volume information and provides healthcare depends on the quality of information available to the doctor or healthcare provider. For example, if the doctor does not know that the patient has diabetes or high blood pressure, the doctor may use some treatments that may lead to deterioration of the condition. The HCIS like any other system have input, operation, and output, and previous definitions of hospital information systems show that these systems are composed of integrated subsystems that interact with one another in an open environment. Thus, the most important of these systems is Patient Electronic Record System, Patient Administrative System, Laboratory Information System, Pharmacy Information System, Radiology System, Picture Achieving, and Communication System and Computerized Physician Order Entry. As well as, for enhancing the quality of patient healthcare by using the IS must use full and accurate documentation of the patient's history, the health and illness that the patient complains, and the treatment plan. Additionally, the quality of healthcare not only based on the availability of medical information but also on the availability of administrative and financial information to provide the required support for internal operations (i.e. the doctor needs to have a good nursing staff and modern medical equipment to implement a good job). Thus, HCIS institution has seven aims as assurance from healthcare quality, productivity improvement, cost management, demand assessment and analysis deployment, facilitate the planning process internally and externally, program assessment and plan, healthcare research and learning. Moreover, the HCIS can reach this aim by implementing the Deming Cycle PDCA which depends on the internal and external customer (hospital staff and patient) they involved into the process to rise acceptance of the result, the figure 1 below describes the implementation mechanism [2]:

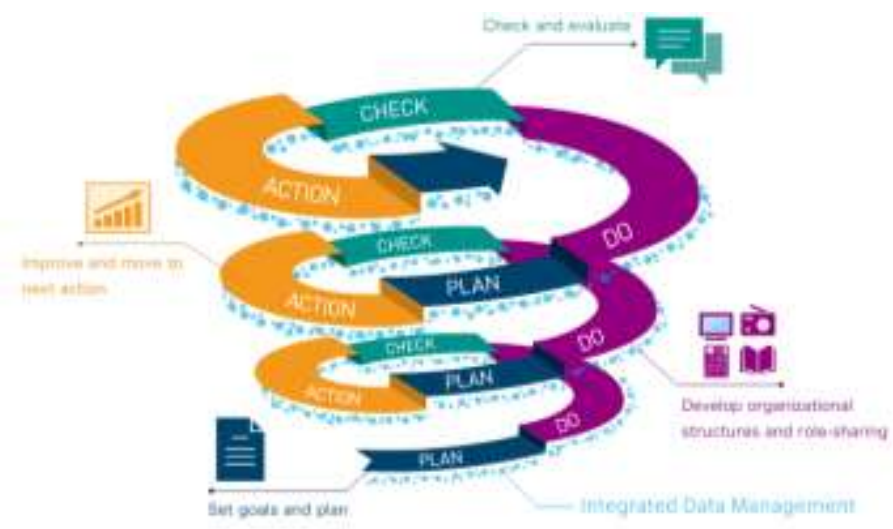

Figure 1: Deming Cycle (PDCA)

To applying the PDCA must implement four steps:

- Plan: Defining a problem present within a process.

- Do: After the planning, it's time to execute the plan.

- Check: Use PDCA in methodology development to track the performance of this result over time.

- Act: Time to standardize the process improvement and implement it across business practices [25].

\section{QUALITY MEASUREMENT}

Healthcare system quality and productivity play a significant role in both the overall society and healthcare system users. The measurement procedure of HCIS quality is a process of using data to assess the performance of health strategies and healthcare providers against documented quality standards. Thus, the evaluation of care extends across the full domain of healthcare settings, from physician' offices, facilities to hospital systems [8]. As well as, a rigorous measurement or evaluation of HCIS is recommended and very important for the provider, decision-makers and users to enhance the TQM, reduce errors, and growth the efficiency of clinical care and its cost. However, the evaluation can be defined as the crucial estimation of identified objects, based on a set of rules, to solve a given problem and achieve the primary goals of an organization [1]. The assessment of HCIS doesn't focus on the technical issues only but also covering human and organizational issues [26]. Therefore, there are several aspects must be using to evaluate the quality level of health care. For example, the resources structure for healthcare system. This structure including the accessibility of services, portability of HCIS, safety, usability, and the security of all parts of the healthcare system. Also, measures the process of care including supervision of diseases, prevention care, the precision of diagnosis, and the accuracy of treatment. The process of care includes the timeliness, provide services, and coordination of care between different departments and disciplines. Finally, the evaluation of healthcare outcomes has a role in quality 
monitoring for healthcare through measure the success of satisfying patient and provider needs and the achievement extent of healthcare objective [15]. In healthcare organization, the information system must be updated, used easily and facilitated the overall work. To evaluate the performance of information system, we need to measure the quality of services that provided for the end users. However, this paper proposed the SERVPERF scale as a service quality measurement that help in assess the quality of services. Obviously, it is contained seven dimensions and each of them has some attributes as follows [14]:

Table 1: HCIS Measurement Dimensions

\begin{tabular}{|c|c|}
\hline Dimension Name & Dimension Elements \\
\hline $\begin{array}{l}\text { 1. System or Website } \\
\text { design }\end{array}$ & $\begin{array}{l}\text { - Implement attractive and efficient system } \\
\text { - } \text { Consistent and institutionalized navigation } \\
\text { - Well-composed appearance of UI } \\
\text { - Execute transaction easily } \\
\text { - } \text { Rabidly update and download }\end{array}$ \\
\hline 2. Reliability & $\begin{array}{l}\text { - Accurate records information } \\
\text { - System available all the time } \\
\text { - Keeping advancement guarantee } \\
\text { - Provide a constant online service (ex. online health consultancy or } \\
\text { appointment reservation) } \\
\text { - Complete request it services } \\
\text { - Healthcare provider being honest about offering services }\end{array}$ \\
\hline 3. Responsiveness & $\begin{array}{l}\text { - Satisfactory contact data and performance } \\
\text { - Prompt reactions to clients } \\
\text { - Timely reactions to clients. } \\
\text { - Adequate reaction time. } \\
\text { - Quickly take care of issues }\end{array}$ \\
\hline 4. Security & $\begin{array}{l}\text { - Ensure from protect clients' information } \\
\text { - Check the integrity of the client data } \\
\text { - Good notoriety }\end{array}$ \\
\hline 5. Fulfillment & $\begin{array}{l}\text { - Ability to modify the records and appointment etc. } \\
\text { - Accurate guarantees about quality of provider services }\end{array}$ \\
\hline 6. Information & $\begin{array}{l}\text { - Refreshed data } \\
\text { - Information present and relevant. } \\
\text { - Information precise and significant } \\
\text { - Information straightforward } \\
\end{array}$ \\
\hline 7. Empathy & $\begin{array}{l}\text { - } \text { Good individual care } \\
\text { - Adequate contacts } \\
\text { - Address grievances cordial } \\
\text { - } \text { Consistently considerate } \\
\end{array}$ \\
\hline
\end{tabular}

From the previous table the attempt to increase the quality of HQIS by using this measurement dimensions will provide long-term benefits to the healthcare organization. Such as, simplifying procedures, improved procedures, operating efficiency and reduction operations reputed. In addition, from healthcare interventions over time and between the service providers, the challenge is to identify the greatest appropriate measurement area, Also, with highlighted the importance universal aspects of health performance measures as a following table [24]:

Table 2: Measurement area

\begin{tabular}{|l|l|}
\hline \multicolumn{1}{|c|}{ Measurement area } & \multicolumn{1}{c|}{ Measures Explanation } \\
\hline Population health & $\bullet$ Collected data on the healthcare system for population \\
\hline Clinical quality and appropriateness of care & $\bullet \begin{array}{l}\text { Patients care and services provider that received lead to } \\
\text { achieving the desire results }\end{array}$ \\
& $\begin{array}{l}\text { Decide the internal process and these activities are } \\
\text { implemented in complete a technological way }\end{array}$ \\
\hline
\end{tabular}




\begin{tabular}{|c|c|}
\hline Responsiveness of health system & $\begin{array}{l}\text { Concerns of patient dignity, independence, privacy, } \\
\text { communication, rapidly care, social support and quality of } \\
\text { elementary facilities. } \\
\text { - Approach and environment that provide the treated way for } \\
\text { the patient to the healthcare system. }\end{array}$ \\
\hline Equity & $\begin{array}{l}\text { The degree of health equity, healthcare system access, } \\
\text { responsiveness and financing }\end{array}$ \\
\hline Productivity & $\begin{array}{l}\text { - Efficiency of the healthcare system, healthcare institution } \\
\text { and individual practitioners }\end{array}$ \\
\hline Outcome & $\begin{array}{l}\text { - Hard to manipulated } \\
\text { - Meaningful for stakeholders } \\
\text { - Enhance the long-term of health advancement strategies } \\
\text { - Care about health goal that related to the patients }\end{array}$ \\
\hline Process & $\begin{array}{l}\text { - Mostly observed easily } \\
\text { - Sensitive in quality care } \\
\text { - Easier to interpret } \\
\text { - Provide clear ways for process } \\
\text { - To find statistically significant effects need to small sample }\end{array}$ \\
\hline
\end{tabular}

Finally, during measure the quality of HCIS several problems reported which pose barriers to its evaluators. For instance, conflicting and modification of the evaluation goals during the assessment process. Secondly, complicated and contradictory results. Thirdly, uncertainty either outcomes can be generalized to another situation. Also, the evaluation result based on users' perspective and expectations. Finally, the assessment of HCIS needs tremendous efforts and time for the establishment and enforcement [1]. Consequently, for a success of evaluation activity, a wellplanned process is recommended with clear identification for the evaluation's objectives that assess by an expert.

\section{THE IMPACT OF HCIS ON QUALITY}

The information system of healthcare environment provides a powerful force to elevate the quality level of the of healthcare and prevents any errors may be happening with nurse, physician, patients and anyone involved in health workflow. The impact of HCIS will appear on many aspects such as, the consultation result of the patient, with HCIS the information becomes more accurate, consistent and timely delivery for valuable clinical knowledge. Also, it will be more corrective by preventing any errors that may horrify the patient in his lher treatment information. The preventing healthcare errors through enhance the patient records, merging the computerized clinic with decision support system, and consider the patient as co-procedure of quality care. The best instance system for applying this impact is used EHR system. Secondly, a portable framework of HCIS which assist to share and manage the health information between patient and medical staff and enhance the health quality workflow process. This feature reduces the number of inpatient/outpatient visits to the hospital location. Furthermore, the web browser is one of the variant ways for achieving the portability function. Based on this feature, the patient can contact directly with physician's office for the variant purpose. Moreover, the portability feature is being tapped by healthcare team in order to publish their initiatives in healthcare domain as health education, health promotion, diseases prevention, and disease management. Thirdly, HCIS provides a tracking system for recording patient state at a time. This facility used to enhance the quality of healthcare from different two sides. The first side belongs to the patient care by tracking the patient over time in order to have a continuous evaluation for the patient state and have a rapid response in the emergency. The EHR is one of tracking system instance that built to enhance the impact of HCIS. On the other side, for working on the health research as epidemiological studies, the tracking system is required in this case. To do that, the information system tracks the population of the patient, register, and analyze all data to evaluate the status and recommend the possible results. Additionally, healthcare organizations are particularly vulnerable to internal and external threats. So, using highsecurity techniques with HCIS will preserve the healthcare assets, safeguard patient records and sensitive data. Thus, the healthcare environment tends to increase its alertness reaction by preventing a disruption or loss by using the backup, redundancy strategies and, enhance the user awareness. Moreover, the integration between finical and clinical data on one IS will enhance the workflow of the healthcare process. Therefore, all tasks that a provider needs for one patient exists on one HCIS that help to preserve the working time with a quick access and retrieve the required information. Consequent for this integration, it leads to increase the healthcare throughputs and the patient satisfaction. Also, decreases the total cost. Additionally, the remotely delivering the healthcare is the most revolutionary facility provide by an information system. Based on this feature, doctors can remotely diagnose, assess and treat the patient. 


\section{IARJSET

This feature not only covering the healthcare in outlying areas but also guarantees the people get a high level of quality at a very reasonable cost. Therefore, the medical staff doesn't have to travel to these remote locations which help to preserve their time and maximize their throughput and effort [4]; [9].

\section{HCIS EXAMPLES}

In order to enhance the TQM of the Healthcare, the number of the HCIS instances rise to achieve the high-quality services. For instance, the Electronic Health Record (EHR) system build to integrate all health services for inpatient and outpatient in one coordinated and integrated system. This system used by both medical staff and patients who act as a significant role in their healthcare process. EHR works to maximize the quality of the healthcare by using many IS facilities as reminder alert, checklists, a predicative tool to avoid the emergency critical situations, electronic prescribing, test ordering within precise communication among the different disciplines. Moreover, it allows the portal access to the patients' records and immediate access to his medical history. These facilities play key roles in quality improvement by reducing the error rate and redundancy, enhance the workflow of patient data, minimize the data and test replication, rapidly respond to patient inquiries, reporting faster, make the care more effective, customize, preserve the time and safer [23]; [13]. In the same token, the quality assessment tool which is Ensuring Quality Information for Patients (EQIP) is a tool that provides an objective and precision way of written healthcare information in order to preserve the consultation information. This tool conducted in three stages which are: (1) defining the presentation quality of written healthcare information; (2) pilot the tool before roll out in order to ensure the criteria validity, (3) using enormous number of different samples to evaluate the utility and the reliability of written healthcare information [17]. Moreover, written patient information must meet keys standards of the quality of the readability, clarity, evidence based, timely, and completeness. Consequently, this tool improves the healthcare quality by minimizes the anxiety, gain the self-care, and the obligation's therapy, prevent the communication problems between health providers, and patients, also maximize the usage of health service in an effective way [22]. Another instance of how IS role improving the healthcare quality is Electronic Clinical Reminders (ECR) system for diabetes and coronary artery diseases. This system exceeds the limitation of paper-based reminders with the capability to provides patients immediately recommended care within the rapid response. Also, the physician has the ability to view the reminder information for one patient synchronized with another including active medical problems, prescription, and the critical situations. This improvement in reminder system done through the integration with workflow process and with EHR system [6]. For enhancing the quality of laboratory data an Electronic Laboratory Information System developed. This system conduct needs assessment and integration with workflow analysis for effective impact on patient care and health monitoring. Further, the laboratory system improves the quality of healthcare from various aspects as minimizing the redundancy and delay of laboratory results, and prevent the lost or error-prone of laboratory data. Additionally, it decreases a remediation time, death-rate, morbidity, and length of hospital lodging. In addition, for providing an integrated healthcare delivery system with use innovative information system and decision support to implement quality assurance actions, the Veterans Health Administration (VHA) an example. This system includes outpatient, inpatient, and longterm of care. Moreover, it supports the local daily operation in facilities with keep focusing into patient care directly. Also, it provides source data for financial, administrative, and capacity databases [12]. Finally, the Electronic Medical Record (EMR) is a digital version of a paper-based system that holds a patient's medical history from one practice. The provider use it to specify the diagnosis and treatment. Also, the EMR information is difficult to share it with provider outside the area. A patient's records have an ability of print out then send it to the specialist and care team. Obviously, there is a different between EMR and EHR the second one share information with all provider with exceed the collected data by provider's office, and contain a more complete patient history. Therefore, the EMR provide benefit with comparing with paper, it tracks the data, specify preventive visits and screenings for a patient, monitor the patient condition and improve the quality of healthcare [11].

\section{SIGNIFICANT RESULTS AND SUGGESTIONS}

According to an overview, the HCIS impact for improving the quality of the healthcare, we reached to the following results.

1. The healthcare organization work within a complicated framework as result of different environmental factors, which effects on their effectiveness regarding the use advanced information systems.

2. The importance of information systems and their impact on the quality of healthcare service appear by the availability of information lead to facilitate the work accomplishment.

3. The using of information systems in activities of the healthcare organization will lead to raising and improving efficiency and achieve its goals and achieve an elevated level of quality.

4. Lack of understanding the importance of information systems will result in poor services quality that healthcare organization provided to patients.

5. The using of information systems greatly helps to get attention for quality management training programs. 


\section{IARJSET

6. Taking care of all the technical aspects of the information, building and implementing an improved performance strategy for different sections in the healthcare organization that improve the high-quality service.

Moreover, in order to improve the HCIS services and meet the patient and provider satisfaction. We will suggest some of functional features. These features will facilitate the reservation process, provide a fast service, reduce the waiting time and organize the procedures of admitting ward, and hospitals in providing their service by the professional. Also, have a fast way that leads to obtaining more customers they promote the level of confidence between them. Thus, the suggestions include the following points:

1. In order to save patients and staffs time, make reservation process more quickly, easy, and flexible, the HCIS will let the patient make registration, search for an appointment, and confirm the consultation fee on the system from their home.

2. Takes patient's fingerprint instead of a signature to ensure information that submitted on the website for improving the paperless HCIS.

3. Export patient's medical card with a uniform barcode to improve the self-serve process.

4. The system will show the patients order in the queue that will give the patients a clear view of waiting in the queue. Also, the receptionist employer will take off from patients' questions pressure.

5. The system will notify the patients when their appointment comes by using display monitor which placed on the different location in a hospital. This feature allows for the patients to go directly towards the doctor's clinic.

6. To remind the appointment, the system will send a notification message for the patients when they didn't come to the hospital according to their appointment.

\section{CONCLUSION AND FUTURE WORK}

The integration between healthcare and information system is very significant to enhance the quality level of patient care. Therefore, this paper provides an overview of the healthcare system in the past, present, and studied the transformation challenges. Moreover, the paper focused on the need of developing and designing the appropriate HCIS for health services to accelerate the quality provision of health services. Additionally, measurements used to increase the quality level of a healthcare environment. Thus, keep improving the information systems and achieving relevance between service delivery and the total quality, with following the affected variables. Consequently, the using of HCIS activities of the healthcare organization will lead to improving efficiency and achieve an elevated level of quality. The lack of understanding the importance of information systems will result in poor services quality that healthcare organization provided to patients. Finally, this study guides us to do a deep research on the Saudi Arabia healthcare environment, to study its HCIS, what are the strongest and weakest points of the system, how it effects on the healthcare quality, and the way of improving the TQM for Saudi's healthcare organization.

\section{REFERENCES}

[1] Elske Ammenwerth, Stefan Gräber, Gabriele Herrmann, Thomas Bürkle, and Jochem König. Evaluation of health information systems problems and challenges. International journal of medical informatics, 71(2):125-135, 2003.

[2] Asahi Advertising Inc ASAKO. Pdca cycle, 2014.

[3] Asbjorn Aune. Quality and quality management at a crossroads. Total Quality Management, 9(4-5):6-12, 1998.

[4] E Andrew Balas. Information systems can prevent errors and improve quality, 2001.

[5] Marc Berg. Implementing information systems in health care organizations: myths and challenges. International journal of medical informatics, 64(2):143-156, 2001.

[6] Joaquin A Blaya, Sonya S Shin, Martin JA Yagui, Gloria Yale, Carmen Z Suarez, Luis L Asencios, J Peter Cegielski, and Hamish SF Fraser. A web-based laboratory information system to improve quality of care of tuberculosis patients in peru: functional requirements, implementation and usage statistics. BMC medical informatics and decision making, 7(1):33, 2007.

[7] Matt-Mouley Bouamrane, Frances Mair, and Cui Tao. An overview of electronic health information management systems quality assessment. In Proceedings of the 2nd international workshop on managing interoperability and complexity in health systems, pages 37-46. ACM, 2012.

[8] Kim Bailey Caitlin Morris. Measuring health care quality: An overview of quality measures. Families USA, 2014.

[9] Ewan B Ferlie and Stephen M Shortell. Improving the quality of health care in the united kingdom and the united states: a framework for change. The Milbank Quarterly, 79(2):281-315, 2001.

[10] Amani Ghazzawi, Amjad Alharbi, and Azrilah Abd Aziz. Tqm in e-commerce: Issues, implementation and challenges. 2016.

[11] Richard Hillestad, James Bigelow, Anthony Bower, Federico Girosi, Robin Meili, Richard Scoville, and Roger Taylor. Can electronic medical record systems transform health care? potential health benefits, savings, and costs. Health affairs, 24(5):1103-1117, 2005.

[12] Denise M Hynes, Ruth A Perrin, Steven Rappaport, Joanne M Stevens, and John G Demakis. Informatics resources to support health care quality improvement in the veterans health administration. Journal of the American Medical Informatics Association, 11(5):344-350, 2004.

[13] Tiffany F Kelley, Debra H Brandon, and Sharron L Docherty. Electronic nursing documentation as a strategy to improve quality of patient care. Journal of nursing scholarship, 43(2):154-162, 2011

[14] Hongxiu $\mathrm{Li}$ and Reima Suomi. A proposed scale for measuring e-service quality. International Journal of u-and e-Service, Science and Technology, 2(1):1-10, 2009.

[15] Pietro Giorgio Lovaglio. Benchmarking strategies for measuring the quality of healthcare: problems and prospects. The Scientific World Journal, 2012, 2012. 
[16] Angel R Martnez-Lorente, Frank Dewhurst, and Barrie G Dale. Total quality management: origins and evolution of the term. The TQM Magazine, 10(5):378-386, 1998.

[17] Beki Moult, Linda S Franck, and Helen Brady. Ensuring quality information for patients: development and preliminary validation of a new instrument to improve the quality of written health care information. Health Expectations, 7(2):165-175, 2004.

[18] Joyce C Niland, Layla Rouse, and Douglas C Stahl. An informatics blueprint for healthcare quality information systems. Journal of the American Medical Informatics Association, 13(4):402-417, 2006.

[19] SHAUN OHANLON. Paperless nhs-how to transform hospital information technology, June 2015.

[20] Patrick Asubonteng Rivers. Aligning information systems for effective total quality management implementation in health care organizations. Total Quality Management, 10(2):281-289, 1999.

[21] SAHI. What is health informatics, 2017 [ONLINE] http://www.sahi.org.sa/?page_id=2375\&lang=eng?

[22] Thomas D Sequist, Tejal K Gandhi, Andrew S Karson, Julie M Fiskio, Donald Bugbee, Michael Sperling, E Francis Cook, E John Orav, David G Fairchild, and David W Bates. A randomized trial of electronic clinical reminders to improve quality of care for diabetes and coronary artery disease. Journal of the American Medical Informatics Association, 12(4):431-437, 2005.

[23] Sharon Silow-Carroll, Jennifer N Edwards, and Diana Rodin. Using electronic health records to improve quality and efficiency: the experiences of leading hospitals. Issue Brief (Commonw Fund), 17:1-40, 2012.

[24] Peter C Smith. Performance measurement for health system improvement: experiences, challenges and prospects. Cambridge University Press, 2009.

[25] M Sokovic, D Pavletic, and K Kern Pipan. Quality improvement methodologies-pdca cycle, radar matrix, dmaic and dfss. Journal of Achievements in Materials and Manufacturing Engineering, 43(1):476-483, 2010.

[26] Maryati Mohd Yusof, Anastasia Papazafeiropoulou, Ray J Paul, and Lampros K Stergioulas. Investigating evaluation frameworks for health information systems. International journal of medical informatics, 77(6):377-385, 2008. 